UCRL-JC-122859

PREPRINT

CONf- $960493-1$

\title{
A Gain-Clamped, Crosstalk Free, Vertical Cavity Lasing Semiconductor Optical Amplifier for WEM Applications
}

J.D. Walker, F.G. Patterson, S.P. Dijaili, and R.J. Deri

s.
RECEIVED

FEB 121996

OSTI

This paper was prepared for submittal to the Integrated Photonics Research Conference

Boston, MA

April 29-May 3, 1996

December 1995

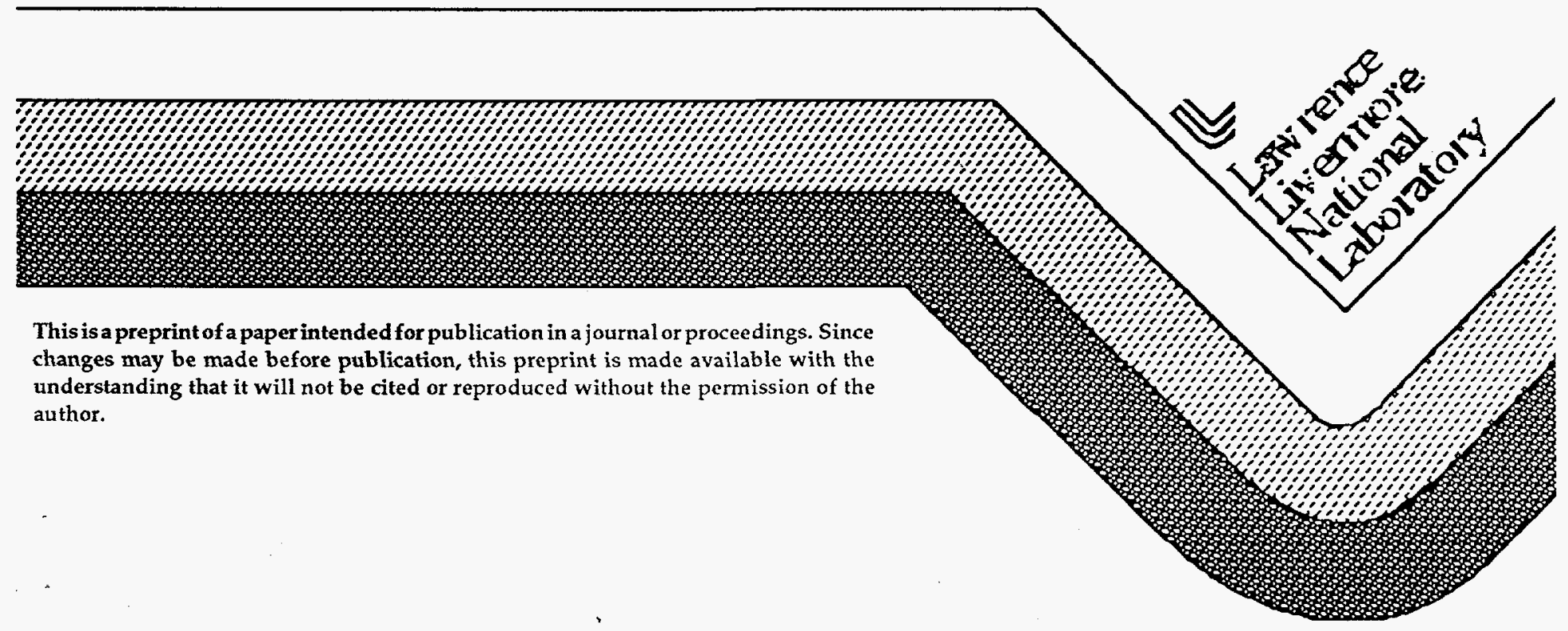




\section{DISCLAIMER}

This document was prepared as an account of work sponsored by an agency of the United States Government. Neither the United States Government nor the University of California nor any of their employees, makes any warranty, express or implied, or assumes any legal liability or responsibility for the accuracy, completeness, or usefulness of any information, apparatus, product, or process disclosed, or represents that its use would not infringe privately owned rights. Reference herein to any specific commercial products, process, or service by trade name, trademark, manufacturer, or otherwise, does not necessarily constitute or imply its endorsement, recommendation, or favoring by the United States Government or the University of California. The views and opinions of authors expressed herein do not necessarily state or reflect those of the United States Government or the University of California, and shall not be used for advertising or product endorsement purposes.

$$
\text { cind }
$$




\section{DISCLAIMER}

Portions of this document may be illegible in electronic image products. Images are produced from the best available original document. 
A GAIN-CLAMPED, CROSSTALK FREE, VERTICAL CAVITY LASING SEMICONDUCTOR OPTICAL AMPLIFIER FOR WPM APPLICATIONS

J.D. Walker, F.G. Patterson, S.P. Dijaili and R. J. Peri'

Lawrence Livermore National Laboratory, PO Box 808, Livermore, CA 94550 tel. (510) 422-3159, fax (510) 422-2783

Abstract

We have fabricated and tested a semiconductor optical amplifier that is gain clamped by vertical cavity lasing and amplifies horizontally with $25 \mathrm{~dB}$ crosstalk suppression. 


\title{
A GAIN-CLAMPED, CROSSTALK FREE, VERTICAL CAVITY LASING SEMICONDUCTOR OPTICAL AMPLIFIER FOR WDM APPLICATIONS
}

\author{
J.D. Walker, F.G. Patterson, S.P. Dijaili and R. J. Deri \\ Lawrence Livermore National Laboratory, PO Box 808, Livermore, CA: 94550 \\ tel. (510) 422-3159, fax (510) 422-2783
}

\section{INTRODUCTION:}

In recent years, advancements in semiconductor optical amplifier (SOA) technology have solved many of the problems (e.g., polarization sensitivity[1] and low saturated output powers[2]) which have prevented their widespread deployment into optical networks. The most elusive and last remaining problem is the large crosstalk between channels in wavelength division multiplexing (WDM) and time division multiplexing (TDM) applications. The crosstalk arises from dynamic gain variations due to gain saturation. The most effective solution to these problems involves the use of a separate and simultaneous stimulated emission field in the SOA. Above threshold, the gain is clamped to the cavity losses and the effective carrier lifetime is significantly reduced due to stimulated emission. As a result, crosstalk, speed, and linearity of the SOA are significantly improved. Previous implementations of this gain-clamping approach to crosstalk reduction use a longitudinal lasing field[3-5] where a distributed Bragg reflector (DBR) or distributed feedback (DFB) laser structure is modified with lower reflectivities and then pumped above threshold. This approach, where laser light is collinear with the amplified signal, has a number of disadvantages. The creative challenge is to find a configuration where an "extra" lasing field can share the same gain medium and not hinder propagation of the signal beam through the device.

Our solution is to use an orthogonal lasing configuration as shown in Fig. 1(a). The orthogonal lasing field is provided by a vertical-cavity surface-emitting laser diode (VCSEL). A single-mode SOA ridge waveguide is formed in the upper VCSEL mirror. We will refer to this structure as a vertically lasing semiconductor optical amplifier (VLSOA). The advantages of a VLSOA over other gain clamped geometries are: 1) No separate spectral filtering of laser light along the fiber input or output is needed. 2) The full SOA gain spectrum is available for use since no wavelength dependent components are in the signal beam path (e.g., DBRs or dichroic coatings). 3) Since the internal power density is very large in a VCSEL, single mode rate equations show that the VLSOA is significantly faster than other gain clamped SOAs. Thus the VLSOA geometry can provide the highest channel speeds for WDM, TDM, and CATV/microwave applications. 4) Because the lasing field is orthogonal to the signal gain path, the device parameters for lasing (e.g. mirror reflectivities) can be independently tailored from SOA gain (e.g. length). As a result, the trade-off between high lasing field power (i.e. effective gain clamping) and gain along the ridge waveguide is eliminated.

The basic physics of the VLSOA device is now briefly discussed. As the device is electrically pumped, the gain of the amplifier increases until the VCSEL reaches lasing threshold. Above threshold, the gain is saturated or clamped to the passive losses (primarily mirror losses). Any further pumping of the medium results in the lasing power increasing. In the case of the VLSOA, there is good spatial overlap between the signal beam along the ridge waveguide and the lasing field in the vertical direction. The clamping of the gain to the passive losses via feedback (historically analogous to the electrical operational amplifier) by a lasing field implies that all the relevant parameters of a laser then apply such as speed of response, photon lifetime, etc. Since the VCSEL mirror reflectivities are relatively stable to variations in temperature, age, or drift in materials parameters, the SOA gain, is also insensitive to these parameters. One benefit of gain clamping is that the WDM crosstalk is greatly reduced. The lasing field acts as a ballast and an input signal modulates the VCSEL power as opposed to a conventional SOA where an input signal modulates the gain. As the amplified signal output power is increased, the gain is clamped and linear until the laser power is exhausted. Hence, the effective SOA saturation output power equals 
the lasing field power. Another benefit of the VLSOA is that the TDM gain recovery time is greatly reduced from $\sim 1$ ns to $\sim 10$ ps as result of stimulated emission from the lasing field., A third, but yet untested benefit, is the possibility of reducing the amplified spontaneous emission (ASE) noise in the ridge waveguide by microcavity noise reduction. Briefly, the ASE enhancement expected in the vertical direction [6] reduces the ASE for the horizontal direction. Stimulated emission is not affected and hence the SOA gain will not be reduced. It may be possible to exploit this effect to build low noise SOAs.

\section{DEVICE FABRICATION:}

An SEM photo of our first generation VLSOA is shown in Fig. 1(b). The device is essentially a tilted-facet ridge waveguide SOA, except that it uses a VCSEL epitaxial wafer in place of a conventional edge-emitting design. The ridge etched into the top Bragg reflector is used for electrical andioptical confinement along the waveguide, and the tilted facets are used to suppress lasing along the amplifier direction. The VCSEL wafer is a molecular beam epitaxy (MBE) grown structure with GaAs/AlGaAs Bragg reflectors and a InGaAs multiple quantum well active region. The wafer used in these experiments produced room temperature CW output powers of $5 \mathrm{~mW}$ when processed into proton-implanted $30 \mu \mathrm{m}$ square vertical cavity lasers [7]. Details of the VCSEL wafer design and epitaxial growth can be found in the literature [7]. Figure 1(b) is a view of the end facet of the VLSOA. The photo shows a $3 \mu \mathrm{m}$ thick top Bragg reflector (20.5 Bragg pairs, $0.14 \mu \mathrm{m}$ period), active region (white stripe) and $4.5 \mu \mathrm{m}$ thick bottom Bragg reflector. The ridge is $9 \mu \mathrm{m}$ wide, and was defined by etching about half way through the top Bragg reflector with a $\mathrm{H}_{2} \mathrm{SO}_{4}: \mathrm{H}_{2} \mathrm{O}_{2}: \mathrm{H}_{2} \mathrm{O}$ based liquid etch. The top of the ridge is electrically contacted with ebeam and electroplated gold, and there is an insulating $\mathrm{SiO}_{2}$ layer under the gold in the valley regions. The ridge stripes run at a seven degree angle to the (100) cleave planes to prevent longitudinal lasing.

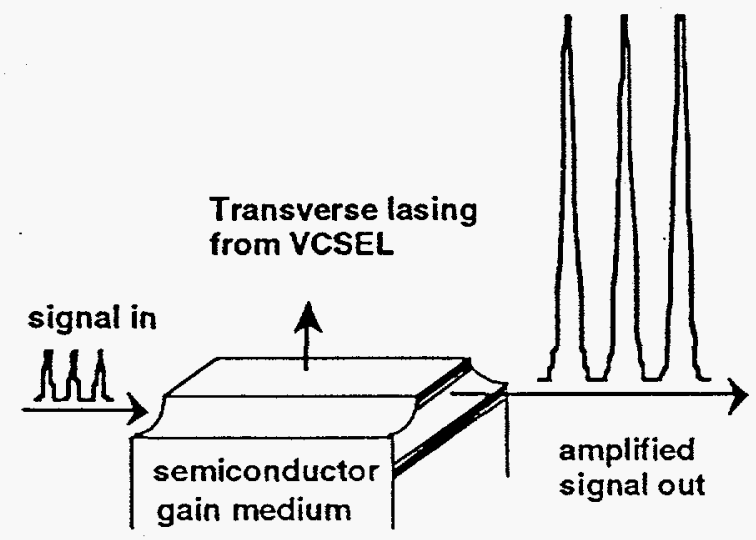

Fig. 1 (a) schematic of the VLSOA

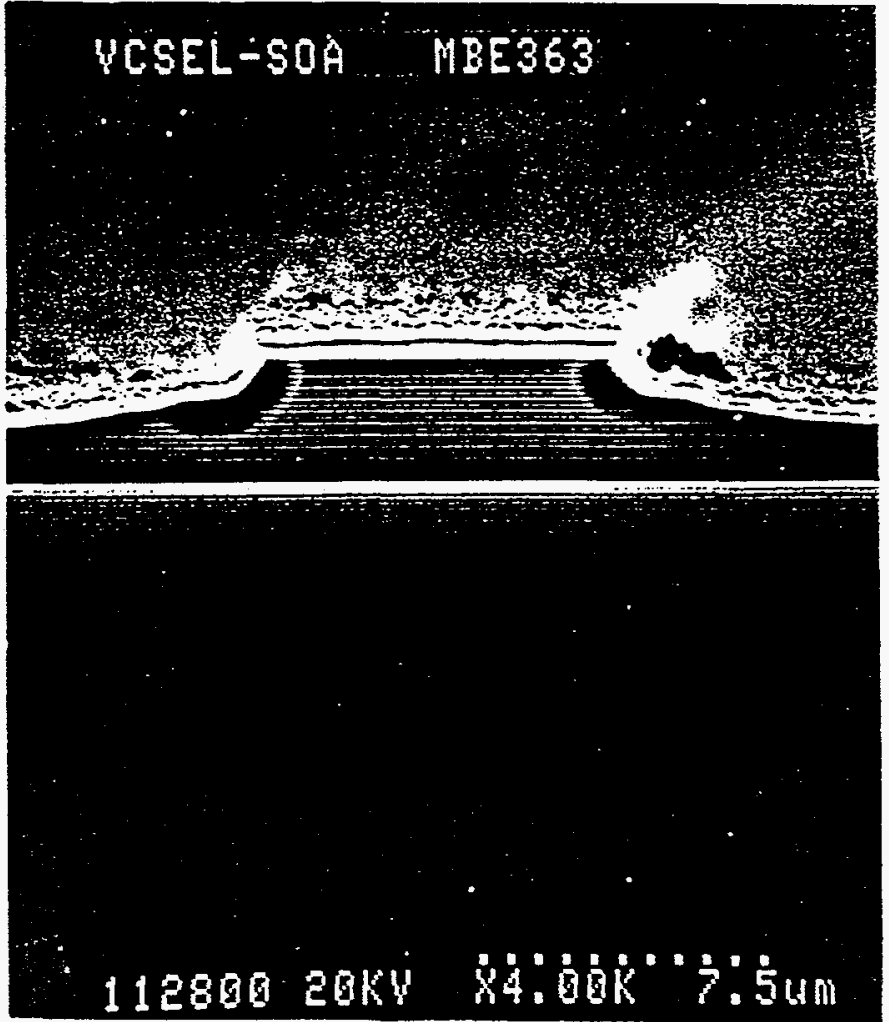

Fig. 1(b) SEM image of VLSOA cleaved facet. 
EXPERIMENTAL RESULTS:
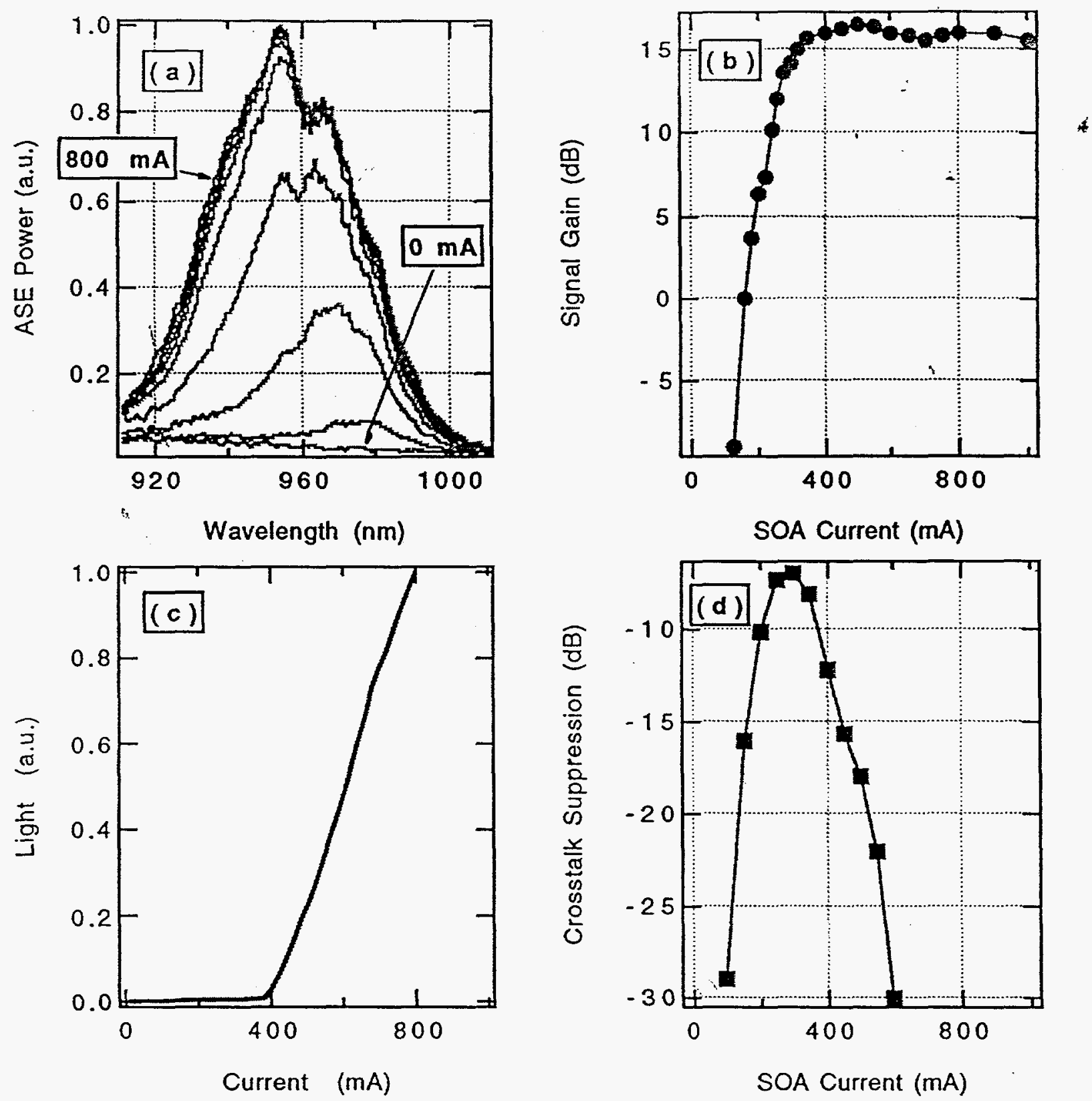

Fig. 2. (a) ASE spectra, $I=0$ to $800 \mathrm{~mA}, 100 \mathrm{~mA}$ steps, res. bandwidth $0.1 \mathrm{~nm}$; (b) VLSOA gain vs. bias current; (c) L-I curve for VCSEL lasing power, (d) VLSOA crosstalk signal vs. bias current

The VLSOAS were cleaved into individual die and mounted active-side up without solder onto a vacuum heat sink. In order to avoid adverse thermal effects, the bias current to the device is pulsed at a $0.2 \%$ duty cycle $(1 \mu \mathrm{s}, 2 \mathrm{kHz})$. (With properly soldered heat sinks $\mathrm{CW}$ operation is possible.) The pulsed L-I curve of the vertical-cavity laser is shown in Fig. 2(c). The VCSEL threshold current is observed to be $400 \mathrm{~mA}$ and thus the VLSOA is expected to be gain-clamped above this current level. The intensity in Fig. 2(c) is in arbitrary units because the devices are covered with gold on both sides and they have no output window to allow detection of the VCSEL light. To obtain the L-I curve, a hole was scratched through the gold on the substrate side of the device to allow some light to escape. The threshold current is higher than VCSEL devices in the literature for two reasons. First, our devices are -100 times larger in area than the low threshold VCSELs in 
the literature, and thus they are expected to have 100 times higher operating currents. Second, our devices have almost no lateral current confinement compared to other VCSEL structures in the literature. Our next generation VLSOA devices will incorporate improved current confinement and should operate $\mathrm{CW}$ at currents comparable to conventional ridge waveguide SOAs $(<100 \mathrm{~mA})$.

The ASE spectra, collected with a single-mode optical fiber from the VLSOA ridge waveguide, are shown as a function of bias current in Fig. 2(a). Nine bias current levels ranging from 0 to 800 : $\mathrm{mA}$ in $100 \mathrm{~mA}$ steps are depicted. For a conventional SOA, the ASE power would continue to increase with bias current up to $800 \mathrm{~mA}$; however, in these VLSOA devices the ASE power is clamped by the internal lasing field when the VCSEL reaches threshold at $400 \mathrm{~mA}$. The second important result from Fig. 2(a) is that no detectable laser light is coupled into the single-mode optical fiber which defines the optical pathway for the amplified signal beam.

Figure 2(b) shows that as the VCSEL reaches threshold, the gain clamps at $16 \mathrm{~dB}$. The gain in this first generation VLSOA is limited by a low and unoptimized mode confinement factor, poor lateral mode control, and low current confinement. These limitations can be overcome in future devices and $30 \mathrm{~dB}$ chip gains should be possible.

The crosstalk signal is measured by introducing a chopped ( $1 \mu \mathrm{s}, 1 \mathrm{kHz})$ Ti:sapphire laser "pump" beam at $960 \mathrm{~nm}$ and a CW laser diode "probe" beam at $954 \mathrm{~nm}$ [8]. A Hewlett-Packard optical spectrum analyzer (OSA) is used to filter and detect only the $954 \mathrm{~nm}$ laser light. A lock-in amplifier is employed to sense the $1 \mathrm{kHz}$ modulation of the pump beam induced onto the probe beam. The crosstalk signal is defined as the ratio of this $1 \mathrm{kHz} \mathrm{AC}$ component divided by the DC magnitude of the transmitted probe beam. The crosstalk signal is shown in Fig. 2(d) as a function of the bias current . A precipitous $25 \mathrm{~dB}$ drop in crosstalk is observed as the current is increased above the $400 \mathrm{~mA}$ VCSEL threshold current, consistent with the onset of gain clamping observed in Figs. 2(a,b,c). For a conventional SOA, the crosstalk signal would continue to increase with bias current up to $800 \mathrm{~mA}$.

\section{CONCLUSION:}

We have proposed, fabricated and tested a vertically lasing semiconductor optical amplifier. The device exhibits a $16 \mathrm{~dB}$ clamped chip gain and $25 \mathrm{~dB}$ reduction in crosstalk relative to the maximum crosstalk which occurs just below the lasing threshold. Virtually no laser light is coupled into either the input or output optical fibers. Short wavelength VCSELs are commercially available and long wavelength VCSEL technology is progressing rapidly [9]. VCSEL epitaxial growth technology is compatible with strained multiple-quantum well material which can be made polarization insensitive [1]. We envision future crosstalk free VLSOAs with polarization insensitivity, low current consumption, high fiber-to-fiber gain, and availability at all major communications wavelengths.

This work was performed under the auspices of the U.S. Department of Energy by Lawrence Livermore National Laboratory under contract No. W-7405-Eng-48. The authors would like to acknowledge the efforts of Richard Combs, Holly Petersen, Bill Goward, and J.S. Smith.

\section{REFERENCES:}

[1] L. F. Tiemeijer et al., Appl. Phys. Lett. 62, 826 (1994).

[2] T. Mamijoh et al., IEEE JQE 30, 524 (1994).

[3] B. Bauer, F. Henry, and R. Shimpe, IEEE PTL 6, 182 (1994).

[4] J. C. Simon et al., Electron. Lett. 30, 49 (1994).

[5] L. F. Tiemeijer, et al., IEEE PTL 7, 284 (1995).

[6] DeMartini et al., Phys. Rev. Lett. 60, 1711 (1988).

[7] J. D. Walker, D. M. Kuchta, and J. S. Smith, Electron. Lett. 29, 239 (1993).

[8] F.G. Patterson, S.P. Dijaili, and R.J. Deri, IPRC, Dana Point, CA, Feb. 1995, IFA3.

[9] D. I. Babic et al., IEEE PTL 7, 1225 (1995). 